\title{
What's hot that the other CLINIC lot got
}

\author{
Mamta Ruparel
}

\section{DOES RISK BASED ENTRY TO LUNG CANCER SCREENING IMPROVE PROGRAMME EFFICIENCY?}

Lung cancer screening has the potential to save lives, though optimum methods for implementation in a cost-effective manner are yet to be determined. The PANCAN study (Lancet Oncol 2017;18:1523-31) was the first screening study that used a lung cancer risk prediction algorithm to determine entry into screening. This single-arm prospective study recruited 2537 individuals aged 50-75 with a $2 \%$ 6-year risk of lung cancer as calculated by the 'PANCAN' risk prediction model, a precursor to the PLCO The investigators report a higher incidence of lung cancers $(6.5 \%)$ compared with the National Lung Screening Trial (NLST, 4\%; $\mathrm{P}<0.001$ ) and a higher proportion of early stage cancers $(77 \%$ vs. $57 \%$ in NLST and $25 \%$ in Canadian non-screening registry data, $\mathrm{P}<0.001)$. This suggests scope for an equivalent or better mortality benefit than that reported in NLST. Another interesting finding is the low rate of cancers in patients with no nodules $(n=1)$ compared with those with nodules $>1 \mathrm{~mm}(\mathrm{n}=85)$ and supports the use of less frequent screening in individuals with no nodules on baseline scan. This study has provided compelling evidence for risk-based entry into lung cancer screening to be superior to age and smoking history alone. Such targeted screening may enhance cost-effectiveness of lung cancer screening programmes.

\section{BAP1: A TARGETABLE BIOMARKER FOR rTRAIL TREATMENT OF MALIGNANT MESOTHELIOMA?}

To date, no agents have shown significant efficacy in the treatment of malignant mesothelioma (MM), though early in vitro and animal studies have shown promising results with recombinant TNF-related apoptosis inducing ligand (rTRAIL). Kolluri and colleagues (eLIFE 2018;7:e30224) report a series of experiments to identify novel therapeutic

Correspondence to Dr Mamta Ruparel, Lungs for Living Research Centre, UCL Respiratory, University College London, London WC1E 6JF, UK; m.rupare|@ucl.ac.uk agents in $\mathrm{MM}$ and elucidate potential mechanisms of action to demonstrate biological plausibility of the association. The investigators used a series of experiments with MM cell lines to screen action of 94 anticancer drugs establishing an association between BRCA associated protein-1 (BAP1) and rTRAIL with subsequent testing. They elucidated the underlying mechanism of action and confirmed their findings using external MM cell lines. The authors have elegantly demonstrated that BAP1, LOF mutations result in modulation of specific proteins that invoke sensitivity to rTRAIL. If these findings are reproduced in human in vivo studies, BAP1 mutations could signify a biomarker for rTRAIL therapy in patients with MM.

\section{CAN SERUM LDH AND FULL BLOOD COUNT DETERMINE LUNG CANCER RESPONSIVENESS TO IMMUNOTHERAPY?}

In patients with non-small-cell lung cancer (NSCLC) who express PDL-1, immune checkpoint inhibitor (IKI) treatment confers a survival advantage, but currently there is a lack of a clinically accessible tool to identify patients likely to respond. Mezquita and colleagues (JAMA Oncology: doi:10.1001/ jamaoncol.2017.4771) used three retrospective datasets to examine the relationship between baseline plasma lactate dehydrogenase $(\mathrm{LDH})$ and derived neutrophil to lymphocyte ratio (dNLR; absolute neutrophil count/(white blood cell concentration - absolute neutrophil count)) with overall survival (OS), progression-free survival (PFS) and response rate (RR). They then used the data to internally and externally validate the LIPI (lung immune prognostic index) score, a composite of dNLR and LDH. In the pooled analysis of the first monocentric and second multicentric cohorts $(n=466)$ of patients treated with IKIs, they found that median OS was 4.8 vs 10.0 vs 16.5 months, and median PFS was 2.0 vs 3.7 vs 6.3 months (both $\mathrm{P}<0.001)$ for the poor, intermediate and good LIPI groups, respectively. LIPI group also correlated with RR. The third cohort $(n=162)$ of patients treated only with standard chemotherapy revealed no significant differences in OS or PFS based on LIPI group and no correlation with RR. In summary, poor baseline LIPI score was correlated with poor outcomes with immunotherapy, but not chemotherapy, suggesting a potential role of LIPI as a cost-effective tool to aid precision medicine in IKI treatment of NSCLC.

\section{DOES OSIMERTINIB IMPROVE SURVIVAL}

IN TREATMENT-NAIVE EGFR-POSITIVE

NON-SMALL-CELL LUNG CANCER

COMPARED WITH 'STANDARD' TYROSINE KINASE INHIBITORS?

Tyrosine kinase inhibitors (TKIs) have become standard therapy for patients with NSCLC with EGFR mutations, extending survival in this patient group. However, resistance to first-generation TKIs limits duration of response. The FLAURA study (NEJM 2018;378:113-25) used a phase III double-blind design to compare osimertinib with standard TKIs (gefitinib or erlotinib). Participants had locally advanced or metastatic EGFR mutation-positive NSCLC and were thus eligible for first-line TKI treatment. The study recruited 556 participants across 132 sites in 29 countries. The primary outcome of PFS was significantly longer in the osimertinib group compared with control (18.9 vs 10.2 months, HR 0.46 , 95\% CI 0.37 to $0.57, \mathrm{P}<0.001)$. Positive secondary outcomes included lower rate of central nervous system progression (6\% vs $15 \%$ ), longer duration of treatment response (17.2 vs 8.5 months) and greater decrease in lesion size $(-54.7 \%$ vs $-48.5 \%, \mathrm{P}=0.003)$. The safety profiles were similar in both groups. This study encouragingly suggests that osimertinib has significant clinical benefits over first-generation TKIs and may further improve survival in this patient group.

Funding The author has not declared a specific grant for this research from any funding agency in the public, commercial or not-for-profit sectors.

\section{Competing interests None declared.}

Provenance and peer review Commissioned; internally peer reviewed.

(c) Article author(s) (or their employer(s) unless otherwise stated in the text of the article) 2018. All rights reserved. No commercial use is permitted unless otherwise expressly granted.

\section{Check for updates}

To cite Ruparel M. Thorax 2018;73:500.

Thorax 2018;73:500.

doi:10.1136/thoraxjnl-2018-211806 